\title{
ON CYCLOTOMIC FACTORS OF POLYNOMIALS RELATED TO MODULAR FORMS
}

\author{
BERNHARD HEIM, FLORIAN LUCA, AND MARKUS NEUHAUSER
}

\begin{abstract}
The Fourier coefficients of powers of the Dedekind eta function can be studied simultaneously. The vanishing of the coefficients varies from super lacunary (Euler, Jacobi identities) and lacunary (CM forms), to non-vanishing (Lehmer conjecture for the Ramanujan numbers). We study polynomials of degree $n$, whose roots control the vanishing of the $n$-th Fourier coefficients of such powers. We prove that every root of unity appearing as any root of these polynomials has to be of order 2 .
\end{abstract}

\section{INTRODUCTION}

In his celebrated paper [7], Serre proved that even powers of the Dedekind eta function $\eta(\tau)^{k}$ are lacunary if and only if

$$
k \in\{2,4,6,8,10,14,26\} .
$$

The Dedekind eta function is a modular form and directly related with infinite products studied by Euler.

$$
\eta(\tau):=q^{\frac{1}{24}} \prod_{n=1}^{\infty}\left(1-q^{n}\right), \quad\left(q:=e^{2 \pi i \tau}, \operatorname{Im}(\tau)>0\right) .
$$

Euler and Jacobi proved that (see also [5], [2])

$$
\begin{aligned}
\prod_{m=1}^{\infty}\left(1-q^{m}\right) & =\sum_{m=-\infty}^{\infty}(-1)^{m} q^{\frac{3 m^{2}+m}{2}}, \\
\prod_{m=1}^{\infty}\left(1-q^{m}\right)^{3} & =\sum_{m=0}^{\infty}(-1)^{m}(2 m+1) q^{\frac{m^{2}+m}{2}} .
\end{aligned}
$$

Which shows that for $k=1$ and $k=3$ many Fourier coefficients are vanishing, actually in the $k$ odd case, $\eta(\tau)^{k}$ is superlacunary [5] if and only if $k=1$ or $k=3$. Not much is known in the general case, different from the exponents considered by Euler, Jacobi, and Serre. We refer to [1] for an overview.

2010 Mathematics Subject Classification. Primary 11D10, 11F20; Secondary 11F30, 11B83, 11P84,11R18.

Key words and phrases. Dedekind eta function, Fourier coefficients, integer-valued polynomials, cyclotomic polynomials. 
In this paper we study the Fourier coefficients simultaneous for all powers of the Dedekind eta function. We define a family of polynomials $P_{m}(X)$ for $m \in \mathbb{N}_{0}$ with interesting properties. Consider the identity

$$
\sum_{m=0}^{\infty} P_{m}(z) q^{m}=\prod_{m \geq 1}\left(1-q^{m}\right)^{-z} \quad(z \in \mathbb{C}) .
$$

The roots of these polynomials dictate the vanishing properties of Fourier coefficients. These polynomials have degree $m$ and $A_{m}(X):=m ! P_{m}(X) \in$ $\mathbb{Z}[X]$ is normalized. It follows also from the definition that $P_{m}(X)$ are integer-valued polynomials.

For example, let $m=6$. Then

$$
P_{6}(X)=X(X+1)(X+10) R(X),
$$

where $R(x)$ is irreducible over $\mathbb{Q}$. Hence the 6-th Fourier coefficients of $\eta^{0}$, $\eta$ and $\eta^{10}$ are zero. All 6-th Fourier coeffients for other integral powers are not zero. Note, due to the Lehmer conjecture the factor $(X+24)$ should never appear. The factors $(X+1)$ and $(X+3)$ appear infinitely times (see formulae (1) and (2)).

The polynomials can be defined also recursively. We put $P_{0}(X):=1$ and define

$$
P_{m}(X)=\frac{X}{m}\left(\sum_{k=1}^{m} \sigma(k) P_{m-k}(X)\right), \quad m \geq 1 .
$$

Here, $\sigma(k)$ denotes the sum of the divisors of $k$. The first ten polynomials appeared the first time in the work of Newman [3] and Serre [7]. The integral roots of $P_{m}(x)$ for $(1 \leq m \leq 10)$ had been determined and used by Serre to prove the lacunary result. To illustrate the complexity of the topic let us record the first ten polynomials. 


$$
\begin{aligned}
P_{1}(X)= & X \\
2 ! P_{2}(X)= & X^{2}+3 X=X(X+3) \\
3 ! P_{3}(X)= & X\left(X^{2}+9 X+8\right) \\
= & X(X+8)(X+1) \\
4 ! P_{4}(X)= & X\left(X^{3}+18 X^{2}+59 X+42\right) \\
= & X(X+14)(3+X)(X+1) \\
5 ! P_{5}(X)= & X\left(X^{4}+30 X^{3}+215 X^{2}+450 X+144\right) \\
= & X(3+X)(X+6)\left(X^{2}+21 X+8\right) ; \\
6 ! P_{6}(X)= & X\left(X^{5}+45 X^{4}+565 X^{3}+2475 X^{2}+3394 X+1440\right) \\
= & X(X+10)(X+1)\left(X^{3}+34 X^{2}+181 X+144\right) \\
7 ! P_{7}(X)= & X\left(X^{6}+63 X^{5}+1225 X^{4}+9345 X^{3}\right. \\
& \left.+28294 X^{2}+30912 X+5760\right) \\
= & X(X+8)(3+X)(X+2)\left(X^{3}+50 X^{2}+529 X+120\right) \\
8 ! P_{8}(X)= & X\left(X^{7}+84 X^{6}+2338 X^{5}+27720 X^{4}+147889 X^{3}\right. \\
& \left.+340116 X^{2}+293292 X+75600\right) \\
= & X(X+6)(3+X)(X+1) \\
& \left(X^{4}+74 X^{3}+1571 X^{2}+9994 X+4200\right) \\
& +2341332 X^{4}+4335596 X^{3}+3032208 X^{2}+524160 X \\
9 ! P_{9}(X)= & X^{9}+108 X^{8}+4074 X^{7}+69552 X^{6}+579369 X^{5} \\
& (X+14)(X+26)(X+4)(3+X)(X+1) \\
& \left.+11744775 X^{3}+491 X+120\right) ; \\
& +36290736 X^{2}+653184049020 X^{4}+57773700 X^{3} \\
= & X(X+1) R(X)
\end{aligned}
$$

Here $R(X)$ is an irreducible polynomial given by

$$
\begin{aligned}
& R(x)=X^{8}+134 X^{7}+6496 X^{6}+147854 X^{5}+1709659 X^{4} \\
& \quad+10035116 X^{3}+28014804 X^{2}+29758896 X+6531840 .
\end{aligned}
$$

In the papers of Newman [3] and Serre [7] it is already mentioned, that the polynomials $P_{m}(X)$ are completely reducible over $\mathbb{Q}$ for $m \leq 4$. This is also true over $\mathbb{R}$ for $m \leq 9$, but not for $m=10,12,14,15,18$ as we have checked. Hence, it is likely that polynomials $P_{m}(X)$ have complex nonreal roots for large values of $m$ and it becomes natural to ask about their distribution in the complex plane.

The initial motivation for this work was the following question:

Does there exist any $m \in \mathbb{N}$, such that $P_{m}(i)=0$ ?

This is equivalent to the existence of polynomials $P_{m}(X)$, such that

$$
\left(X^{2}+1\right) \mid P_{m}(X) .
$$

Considering $i$ as a root of unity, what about the values $P_{m}(\zeta)$ for roots of unity $\zeta$ of general order $N$ ? Note that in the case $N=2$ due to Euler we 
already have that

$$
(X+1) \mid P_{m}(X) \text { for infinitely many } m \text {. }
$$

Let $N$ be a natural number. Let $\Phi_{N}(X)$ be the $N$-th cyclotomic polynomial:

$$
\Phi_{N}(X):=\prod_{\substack{1 \leq k \leq N \\(k, N)=1}}\left(X-e^{2 \pi i k / N}\right)
$$

The degree of $\Phi_{N}(X)$ is given by the value of the Euler totient function $\varphi(N)$. It is known that this is irreducible. See [8] as a reference for basic properties of cyclotomic polynomials and their splitting fields. Our main result is the following:

Theorem 1. There is no pair of positive integers $(N, m)$ with $N \geq 3$ such that $\Phi_{N}(X) \mid P_{m}(X)$.

The theorem is equivalent to $P_{m}(\zeta) \neq 0$ for any root of unity $\zeta$ of order $N \geq 3$.

It is maybe worth to mention, that although the proof does not reveal much about the distribution of the roots of $P_{m}(X)$ in the complex plane, it reveals a very interesting property of these roots modulo $p$ for every prime number $p$. Namely, it shows that if $m=p \ell+r$, where $\ell=\lfloor m / p\rfloor$ and $r=m-p\lfloor m / p\rfloor \in\{0,1, \ldots, p-1\}$, then

$$
A_{m}(X) \equiv Q_{r, p}(X)\left(X\left(X^{p-1}-1\right)\right)^{\ell} \quad(\bmod p),
$$

where $Q_{r, p}(X)$ is a polynomial of degree $r$. In particular, the roots of $A_{m}(X)$ modulo $p$ are always among the roots of

$$
X\left(X^{p-1}-1\right) \prod_{1 \leq r \leq p-1} Q_{r}(X)
$$

a polynomial of bounded degree $p(p+1) / 2$. Furthermore, the splitting field of $A_{m}(X)$ over the finite field $\mathbb{F}_{p}$ with $p$ elements is of degree at most $p-1$ no matter how large $m$ is. This is certainly a very surprising phenomenon and we do not have an explanation for such regularity.

The polynomials $Q_{r, p}(X)$ play an important role in our proof. Our proof proceeds to show that if there is $N \geq 3$ such that $P_{m}(\zeta)=0$ for some root of unity $\zeta$ of order $N$, then $N$ must be even. Then a multiple of 3 . Then of 5 . And so on, which of course is impossible. The proof proceeds by induction. For the induction step, we need to show that if $p$ is a prime and $q \mid N$ for all primes $p<q$, then also $p \mid N$. For this, we show that none of the polynomials $Q_{r, p}(X)(\bmod p)$ has an irreducible factor of degree $d$ such that $p^{d}-1$ is a multiple of $N$. When $p$ is small $(p \leq 11)$, we show this by computing all polynomials $Q_{r, p}(X)$ and their irreducible factors modulo $p$. For $p \geq 13$, we appeal to general methods of analytic number theory (for $p \geq 5 \times 10^{9}$ ). For example we involve results of Rosser and Schoenfeld 
[6] on certain Chebyshev functions. Finally a computation for $p$ in the intermediary range $\left[13,5 \cdot 10^{9}\right]$ proves our theorem.

\section{Preliminary Results}

From now on, $N \geq 3$ is an integer and $\zeta$ is a root of unity of order $N$. Throughout the paper $p$ and $q$ are prime numbers (except for $q:=e^{2 \pi i \tau}$ in the first part of the introduction).

Lemma 1. Let $Q(X) \in \mathbb{Z}[X]$. Let $p$ be a prime and $\zeta$ be a root of unity of order $N \geq 3$. Assume that $p, k, a, M_{1}, \ldots, M_{k}$ are positive integers, such that:

(i) $p \nmid N$;

(ii) $N \nmid M_{i}$ for $i=1, \ldots, k$;

(iii) Modulo $p$ we have $Q(X) \mid\left(X\left(X^{M_{1}}-1\right) \cdots\left(X^{M_{k}}-1\right)\right)^{a}$.

Then, $Q(\zeta) \neq 0$.

Proof. Condition (iii) tells us that

$$
\left(X\left(X^{M_{1}}-1\right) \cdots\left(X^{M_{k}}-1\right)\right)^{a}=Q(X) R(X)+p S(X)
$$

for some polynomials $R(X), S(X) \in \mathbb{Z}[X]$. Assuming that $Q(\zeta)=0$, we evaluate equation (6) in $X=\zeta$ getting

$$
\left(\zeta\left(\zeta^{M_{1}}-1\right) \cdots\left(\zeta^{M_{k}}-1\right)\right)^{a}=p S(\zeta) .
$$

The algebraic integer $\zeta_{i}:=\zeta^{M_{i}}$ is a root of unity of order

$$
N_{i}=N / \operatorname{gcd}\left(N, M_{i}\right)>1
$$

for $i=1, \ldots, k$ by condition (ii). Taking norms over $\mathbb{K}=\mathbb{Q}(\zeta)$, we get

$$
\left(N_{\mathbb{K} / \mathbb{Q}}(\zeta)\right)^{a} \prod_{i=1}^{k}\left(N_{\mathbb{K} / \mathbb{Q}}\left(\zeta_{i}-1\right)\right)^{a}=N_{\mathbb{K} / \mathbb{Q}}(p S(\zeta)) .
$$

In the left-hand side of $(8)$, we have $N_{\mathbb{K} / \mathbb{Q}}(\zeta)= \pm 1$, and

$$
N_{\mathbb{K} / \mathbb{Q}}\left(\zeta_{i}-1\right)= \pm\left(\Phi_{N_{i}}(1)\right)^{\varphi(N) / \varphi\left(N_{i}\right)}, \quad \text { for } \quad i=1, \ldots, k .
$$

Hence, we get

$$
\pm \prod_{i=1}^{k} \Phi_{N_{i}}(1)^{a_{i}}=p^{\varphi(N)} S,
$$

where $a_{i}=a \varphi(N) / \varphi\left(N_{i}\right)$ for $i=1, \ldots, k$ and $S=N_{\mathbb{K} / \mathbb{Q}}(S(\zeta))$ is an integer. The above relation is impossible since the left-hand side is divisible only by primes dividing $N_{i}$ for $i=1, \ldots, k$; hence, $N$, whereas by (i), $p$ is not a factor of $N$. Here, we used the well-known fact that for every integer $m>1, \Phi_{m}(1)$ is an integer whose prime factors divide $m$.

Further we need the following fact. 
Lemma 2. If $p \geq 2$ is prime, then

$$
A_{p}(X) \equiv X\left(X^{p-1}-1\right) \quad(\bmod p) .
$$

Proof. Note that $P_{m}(x)$ is an integer valued polynomial. Hence,

$$
p ! P_{p}(k) \equiv 0 \quad(\bmod p)
$$

for all $k \in \mathbb{Z}$. It follows that the polynomial $A_{p}(X)$ has roots modulo $p$ at all positive integers $k$. Hence, all residue classes modulo $p$ are roots of $A_{p}(X)$. Since $A_{p}(X)$ is monic of degree $p$, it follows that

$$
A_{p}(X) \equiv \prod_{k=0}^{p-1}(X-k) \equiv X\left(X^{p-1}-1\right) \quad(\bmod p) .
$$

\section{The STRATEGy of The PRoOF}

Let $A_{m}(X)=m ! P_{m}(X)$, then $A_{0}(X)=1, A_{1}(X)=X$, and

$$
A_{m}(X)=X\left(\sum_{k=1}^{m} \sigma(k)(m-1) \cdots(m-k+1) A_{m-k}(X)\right), \quad m \geq 2 .
$$

In particular, $A_{m}(X) \in \mathbb{Z}[X]$. Let us look at $A_{m}(X)$ modulo 2. Since $\sigma(2)=3 \equiv 1(\bmod 2)$ and $2 \mid m(m-1)$ for all $m \geq 1$, we only have the recurrence

$$
A_{m}(X) \equiv X\left(A_{m-1}(X)+(m-1) A_{m-2}(X)\right) \quad \text { for all } \quad m \geq 1 .
$$

In particular, if $m$ is odd then $2 \mid m-1$ and

$$
A_{m}(X) \equiv X A_{m-1}(X) \quad(\bmod 2)
$$

while if $m$ is even then

$$
A_{m}(X) \equiv X\left(A_{m-1}(X)+A_{m-2}(X)\right) \equiv X(X-1) A_{m-2}(X) \quad(\bmod 2) .
$$

In particular, writing $m=2 \ell+r, \ell=\lfloor m / 2\rfloor, r=m-2\lfloor m / 2\rfloor$, and putting $Q_{0}(X):=1, Q_{1}(X):=X$, we get that

$$
\begin{aligned}
A_{m}(X) & \equiv A_{2 \ell+r}(X) \equiv Q_{r}(X) A_{2 \ell}(X) \\
& \equiv Q_{r}(X)(X(X-1)) A_{2(\ell-1)}(X) \equiv \cdots \\
& \equiv Q_{r}(X)(X(X-1))^{\ell} A_{0}(X) \equiv X^{r+\lfloor m / 2\rfloor}(X-1)^{\lfloor m / 2\rfloor \quad(\bmod 2) .} .
\end{aligned}
$$

Assume now that $P_{m}(\zeta)=0$ for some root of unity $\zeta$ of order $N>1$. Then $A_{m}(\zeta)=0$. Assuming that $N$ is odd, we have that $N \geq 3$. Lemma 1 with $Q(X)=A_{m}(X), p=2, a=r+\lfloor m / 2\rfloor, k=1, M_{1}=1$ gives a contradiction. Hence, $2 \mid N$.

Let us record this.

Lemma 3. If $P_{m}(\zeta)=0$ for some $m \geq 1$ and root of unity $\zeta$ of order $N \geq 3$, then $N$ is even. 
There is nothing special about the prime $p=2$ in the above argument. Let's try the prime $p=3$. That is, we reduce the recurrence for the sequence of general term $A_{m}(X)$ modulo 3 . Since $3=\sigma(2)$, and $3 \mid(m-1)(m-2)(m-$ 3) for all $m \geq 3$, we get that

$$
A_{m}(X) \equiv X\left(A_{m-1}(X)+4(m-1)(m-2) A_{m-3}(X)\right) \quad(\bmod 3), \quad m \geq 2 .
$$

In particular,

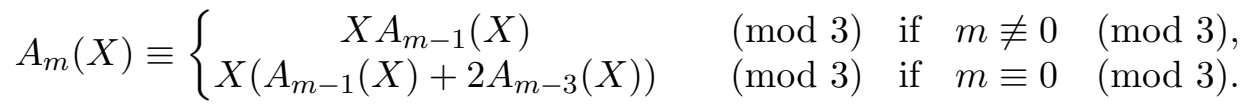

We then get

$$
\begin{aligned}
A_{3 \ell+1}(X) & \equiv X A_{3 \ell}(X) \quad(\bmod 3) \\
A_{3 \ell+2}(X) & \equiv X A_{3 \ell+1}(X) \equiv X^{2} A_{3 \ell}(X) \quad(\bmod 3), \\
A_{3 \ell+3}(X) & \equiv X\left(A_{3 \ell+2}(X)+2 A_{3 \ell}(X)\right) \quad(\bmod 3) \\
& \equiv X\left(X^{2}-1\right) A_{3 \ell}(X) \quad(\bmod 3) .
\end{aligned}
$$

Recursively, we get that if we put $Q_{0}(X):=1, Q_{1}(X):=X, Q_{2}(X):=X^{2}$, $m=3 \ell+r, \ell=\lfloor m / 3\rfloor, r=m-3\lfloor m / 3\rfloor \in\{0,1,2\}$, then

$$
\begin{aligned}
A_{m}(X) & \equiv Q_{r}(X) A_{3 \ell}(X) \equiv Q_{r}(X)\left(X\left(X^{2}-1\right)\right)^{2} A_{3 \ell-3}(X) \equiv \cdots \\
& \equiv Q_{r}(X)\left(X\left(X^{2}-1\right)\right)^{\ell} \quad(\bmod 3) .
\end{aligned}
$$

Hence,

$$
A_{m}(X) \equiv X^{r+\lfloor m / 3\rfloor}\left(X^{2}-1\right)^{\lfloor m / 3\rfloor} \quad(\bmod 3) .
$$

Assume now that $P_{m}(\zeta)=0$ for some root of unity $\zeta$ of order $N$. Then $A_{m}(\zeta)=0$. Assume $3 \nmid N$. Lemma 1 with $Q(X)=A_{m}(X), p=3, a=$ $r+\lfloor m / 3\rfloor, k=1, M_{1}=2$ gives a contradiction. Note that $N \nmid M_{1}$ because $N \geq 4$ (since $N \geq 3$ is even). This contradiction shows that $3 \mid N$.

Let us record what we proved.

Lemma 4. If $P_{m}(\zeta)=0$ for some $m \geq 1$ and root of unity $\zeta$ of order $N \geq 3$, then $3 \mid N$.

Let us continue for a few more steps. We now take $p=5$ and consider the recurrence for $A_{m}(X)$ modulo 5. As before, we obtain the recursion formula:

$$
\begin{aligned}
A_{m}(X) \equiv & X\left(A_{m-1}(X)+3(m-1) A_{m-2}(X)\right. \\
& +4(m-1)(m-2) A_{m-3}(X) \\
& +7(m-1)(m-2)(m-3) A_{m-4}(X) \\
& \left.+6(m-1)(m-2)(m-3)(m-4) A_{m-5}(X)\right) \quad(\bmod 5) .
\end{aligned}
$$


Treating the cases $m=5 \ell+r, r \in\{1,2,3,4,5\}$, we get

$$
\begin{aligned}
A_{5 \ell+1}(X) & \equiv X A_{5 \ell}(X) \quad(\bmod 5) ; \\
A_{5 \ell+2}(X) & \equiv\left(X^{2}+3 X\right) A_{5 \ell}(X) \equiv X(X+3) A_{5 \ell}(X) \quad(\bmod 5) ; \\
A_{5 \ell+3}(X) & \equiv X\left(X^{3}+4 X^{2}+3 X\right) A_{5 \ell}(X) \\
& \equiv X(X+1)(X+3) A_{5 \ell}(X) \quad(\bmod 5) ; \\
A_{5 \ell+4}(X) & \equiv X\left(X^{3}+3 X^{2}+4 X+2\right) A_{5 \ell}(X) \\
& \equiv X(X+1)(X+3)(X+4) A_{5 \ell}(X) \quad(\bmod 5) ; \\
A_{5 \ell+5}(X) & \equiv\left(X\left(X^{4}-1\right)\right) A_{5 \ell}(X) \quad(\bmod 5) .
\end{aligned}
$$

Thus, putting

$$
\begin{aligned}
& Q_{0}(X)=1, \quad Q_{1}(X)=X, \quad Q_{2}(X)=X(X+3), \\
& Q_{3}(X)=X(X+1)(X+3), \quad Q_{4}(X)=X(X+1)(X+3)(X+4),
\end{aligned}
$$

we have that if we write

$$
r=m-5\lfloor m / 5\rfloor \in\{0,1,2,3,4\},
$$

then

$$
A_{m}(X) \equiv Q_{r}(X)\left(X\left(X^{4}-1\right)\right)^{\lfloor m / 5\rfloor}(\bmod 5) .
$$

Note that $Q_{r}(X) \mid X\left(X^{4}-1\right)$. Assume now that $5 \nmid N$. We then apply Lemma 1 with $Q(X)=A_{m}(X), p=5, a=\lfloor m / 5\rfloor+1, k=1, M_{1}=4$ and note that $N \nmid M_{1}$ since $N \geq 6$ (because $N$ is a multiple of 6 ), and we obtain a contradiction.

Let us record what we proved.

Lemma 5. If $P_{m}(\zeta)=0$ for some $m \geq 1$ and root of unity $\zeta$ of order $N$, then $5 \mid N$.

We apply the same program for $p=7$. We skip the details and only show the results. For $r \in\{0,1,2,3,4,5,6\}$, we get

$Q_{0}(X)=1, \quad Q_{1}(X)=X, \quad Q_{2}(X)=X(X+3), \quad Q_{3}(X)=X(X+1)^{2}$, $Q_{4}(X)=X^{2}(X+1)(X+3), \quad Q_{5}(X)=X(X+3)(X+6)\left(X^{2}+1\right)$,

$Q_{6}(X)=X(X+1)(X+3)\left(X^{3}+6 X^{2}+6 X+4\right)$,

where the factors shown above are irreducible modulo 7 . Since $X^{2}+1 \mid X^{4}-1$ and $X^{3}+6 X^{2}+6 X+4 \mid X^{7^{3}-1}-1$, and every root of $Q_{r}(X)$ is of multiplicity at most 2 , it follows that

$$
Q_{r}(X) \mid\left(X\left(X^{6}-1\right)\left(X^{4}-1\right)\left(X^{342}-1\right)\right)^{2} .
$$

Further, writing $m=7 \ell+r$, where $\ell=\lfloor m / 7\rfloor$ and $r=m-7\lfloor m / 7\rfloor$, we get that

Thus, modulo 7 ,

$$
A_{m}(X) \equiv Q_{r}(X)\left(X\left(X^{6}-1\right)\right)^{\lfloor m / 7\rfloor} \quad(\bmod 7)
$$

$$
A_{m}(X) \mid\left(X\left(X^{4}-1\right)\left(X^{6}-1\right)\left(X^{342}-1\right)\right)^{a},
$$

where $a=\lfloor m / 7\rfloor+2$. Assume now that $7 \nmid N$. We apply Lemma 1 with $Q(X)=A_{m}(X), p=7, a=\lfloor m / 7\rfloor+2, k=3, M_{1}=4, M_{2}=6, M_{3}=342$. 
Since $30 \mid N$, it follows that $N \nmid M_{i}$ for $i=1,2,3$. Lemma 1 gives a contradiction.

Thus, we proved the following.

Lemma 6. If $P_{m}(\zeta)=0$ for some $m \geq 1$ and root of unity $\zeta$ of order $N \geq 3$, then $7 \mid N$.

For $p=11$, we have

$$
\begin{aligned}
Q_{0}(X) & =1, \quad Q_{1}(X)=X, \quad Q_{2}(X)=X(X+3), \\
Q_{3}(X) & =X(X+1)(X+8), \\
Q_{4}(X) & =X(X+1)(X+3)^{2}, \\
Q_{5}(X) & =X(X+3)(X+6)\left(X^{2}+10 X+8\right), \\
Q_{6}(X) & =X(X+1)(X+10)\left(X^{3}+X^{2}+5 X+1\right), \\
Q_{7}(X) & =X(X+2)(X+3)(X+8)(X+9)\left(X^{2}+8 X+6\right), \\
Q_{8}(X) & =X(X+1)(X+3)(X+6)(X+10)\left(X^{3}+9 X^{2}+7 X+2\right), \\
Q_{9}(X) & =X(X+1)(X+3)^{2}(X+4)^{2}(X+10)\left(X^{2}+6 X+1\right), \\
Q_{10}(X) & =X(X+1)(X+8)\left(X^{7}+5 X^{6}+10 X^{5}+6 X^{3}+10 X^{2}+X+5\right) .
\end{aligned}
$$

All factors shown are irreducible modulo 11 . We note that the multiplicity of any root of $Q_{r}(X)$ is at most 2. Further, the irreducible factors of the above polynomials which are not linear are of of degrees 2,3 , or 7 over $\mathbb{F}_{11}$.

Hence,

$$
Q_{r}(X) \mid\left(X\left(X^{11-1}-1\right)\left(X^{11^{2}-1}-1\right)\left(X^{11^{3}-1}-1\right)\left(X^{11^{7}-1}-1\right)\right)^{2} .
$$

Writing $m=11 \ell+r$ with $r \in\{0,1, \ldots, 10\}$, where $\ell=\lfloor m / 11\rfloor$, we get that

$$
A_{m}(X) \equiv Q_{r}(X)\left(X\left(X^{10}-1\right)\right)^{\lfloor m / 11\rfloor} \quad(\bmod 11),
$$

so modulo $11, A_{m}(X)$ divides

$$
\left(X\left(X^{10}-1\right)\left(X^{11^{2}-1}-1\right)\left(X^{11^{3}-1}-1\right)\left(X^{11^{7}-1}-1\right)\right)^{a},
$$

where $a=\lfloor m / 11\rfloor+2$. Assume now that $11 \nmid N$. Then we apply Lemma 1 with $Q(X)=A_{m}(X), p=11, a=\lfloor m / 11\rfloor+2, k=4, M_{1}=11-1=$ $10, M_{2}=11^{2}-1=120, M_{3}=11^{3}-1=1330, M_{4}=11^{7}-1=19487170$. Since $2 \cdot 3 \cdot 5 \cdot 7 \mid N$, we get that $N \nmid M_{i}$ for $i=1,2,3,4$. Now Lemma 1 yields to a contradiction.

Thus, we record what we proved.

Lemma 7. If $P_{m}(\zeta)=0$ for some $m \geq 1$ and root of unity $\zeta$ of order $N \geq 3$, then $11 \mid N$.

\section{The CASE OF the General PRIMe $p$}

Assume now that $p \geq 13$ and that we proved that $q \mid N$ holds for all primes $q<p$. Recall that $N \geq 3$ is the order of a root of unity $\zeta$ which is 
also a root of $P_{m}(X)$ for some $m \geq 1$. We would like to prove that $p \mid N$. For this, we compute for $r \in\{0, \ldots, p-1\}$,

$$
Q_{r}(X) \equiv \prod_{i=1}^{s_{r}} Q_{r, i}(X)^{\alpha_{r, i}} \quad(\bmod p),
$$

where $Q_{r, i}(X)$ are distinct irreducible factors of $Q_{r}(X)$ modulo $p$. Assume $Q_{r, i}(X)$ is of degree $d_{r, i}$. Let

$$
\mathcal{D}_{p}=\left\{d_{r, i}: 1 \leq i \leq s_{r}, 1 \leq r \leq p-1\right\} .
$$

Let $\alpha=\max \left\{\alpha_{r, i}: 1 \leq i \leq s_{r}, 1 \leq r \leq p-1\right\}$.

Then, writing $m=p \ell+r$ with $r \in\{0,1, \ldots, p-1\}$, we have

$$
A_{m}(X) \equiv Q_{r}(X)\left(A_{p}(X)\right)^{\ell} \quad(\bmod p) .
$$

This follows by induction from the recursion formula

$$
\begin{aligned}
A_{p \ell+r}(X) & \equiv X\left(\sum_{k=1}^{r} \sigma(k)(p \ell+r-1) \cdots(p \ell+r-k+1) A_{p \ell+r-k}(X)\right) \\
& \equiv X\left(\sum_{k=1}^{r} \sigma(k)(r-1) \cdots(r-k+1) A_{r-k}(X)\right)\left(A_{p}(X)\right)^{\ell} \\
& \equiv A_{r}(X)\left(A_{p}(X)\right)^{\ell} \quad(\bmod p) .
\end{aligned}
$$

By using Lemma 2 we thus get that

$$
A_{m}(X) \equiv Q_{r}(X)\left(X\left(X^{p-1}-1\right)\right)^{\lfloor m / p\rfloor}(\bmod p) .
$$

Hence modulo $p, A_{m}(X)$ divides

$$
\left(X \prod_{d \in \mathcal{D}_{p}}\left(X^{p^{d}-1}-1\right)\right)^{a}
$$

where we can take $a:=\lfloor m / p\rfloor+\alpha$.

Assume that $p \nmid N$. We can then apply Lemma 1 with $Q(X)=A_{m}(X)$, the prime $p$, the number $a, k=\# \mathcal{D}_{p}$ and $M_{j}=p^{d_{j}}-1$ for $j=1, \ldots, k$, where $\mathcal{D}_{p}=\left\{d_{1}, \ldots, d_{k}\right\}$. We need to ensure that $N \nmid M_{j}$ for all $j=1, \ldots, k$. We know that $\prod_{q<p} q \mid N$. Thus, it suffices to show that $\prod_{q<p} q$ is not a divisor of $M_{j}$ for any $j=1, \ldots, k$. Until now, namely for the primes $p \in\{2,3,5,7,11\}$, we checked that this was case by case. To complete the induction, it suffices to show the following lemma.

Lemma 8. If $p \geq 13$, there does not exist a positive integer $1 \leq d \leq p-1$ such that

$$
p^{d}-1 \equiv 0 \quad\left(\bmod \prod_{q<p} q\right)
$$


Assume that we proved the lemma. The above argument shows that if $q \mid N$ for all $q<p$ and $p \geq 13$, then $p \mid N$. Replacing $p$ by the following prime, we get, by induction, that $N$ is divisible by all possible primes, which is a contradiction.

So, it suffices to prove Lemma 8 . This will be proven by analytic methods.

\section{The CASE OF The LARGE PRIME $p$}

Assume $p \geq 13$ and for some $d \leq p-1$, we have $q \mid p^{d}-1$ for all primes $q<p$. Then $d$ is divisible by the $o_{q}(p)$, which is the order of $p$ modulo $q$. We split $q<p$ into two subsets:

$$
Q_{1}=\left\{q<p: o_{q}(p) \leq p^{1 / 2}\right\}, \quad Q_{2}=\left\{q<p: o_{q}(p)>p^{1 / 2}\right\} .
$$

For $Q_{1}$, we have

$$
\prod_{q \in Q_{1}} q \mid \prod_{\substack{e \mid d \\ e \leq p^{1 / 2}}}\left(p^{e}-1\right)
$$

The above leads to

$$
\sum_{q \in Q_{1}} \log q<\sum_{\substack{e \mid d \\ e \leq p^{1 / 2}}} \log \left(p^{e}-1\right)<\log p \sum_{\substack{e \mid d \\ e \leq p^{1 / 2}}} e \leq p^{1 / 2} \tau_{1}(d) \log p .
$$

Here and in what follows we use $\tau_{1}(d)$ for the number of divisors of $d$ which are $\leq p^{1 / 2}$. For $Q_{2}$, let $e \mid d$ with $e>p^{1 / 2}$ and assume that $q \leq p-1$ is such that $o_{p}(q)=e$. Then $e \mid q-1$. Thus, $q \equiv 1(\bmod e)$. Since $q \leq p-1$, it then follows, by counting the number of positive integers less than or equal to $p-1$ which are larger than 1 in the arithmetic progression $1(\bmod e)$ and even ignoring the information that they should also be prime, it follows that the number of choices for such $q$ is at most $(p-1) / e<p^{1 / 2}$. This was for a fixed divisor $e$ of $d$ which exceeds $p^{1 / 2}$. Thus,

$$
\sum_{q \in Q_{2}} \log q \leq p^{1 / 2}\left(\sum_{\substack{e \mid d \\ e>p^{1 / 2}}} 1\right) \log p<p^{1 / 2} \tau_{2}(d) \log p,
$$

where $\tau_{2}(d)$ is the number of divisors of $d$ which are $>p^{1 / 2}$. Thus letting $\theta$ be the Chebyshev function defined for a positive real number $x$ by

$$
\theta(x):=\sum_{q \leq x} \log q
$$

we get

$$
\theta(p)=\sum_{q \leq p} \log q \leq\left(p^{1 / 2} \tau(d)+1\right) \log p,
$$


where $\tau(d)=\tau_{1}(d)+\tau_{2}(d)$ is the total number of divisors of $d$. Assume now that $p>10^{9}$. Theorem 6 in [6] shows that

$$
\theta(p)>0.99 p \text {. }
$$

Further,

$$
\frac{\tau(d)}{d^{1 / 3}}=\prod_{q^{\alpha_{q}} \| d}\left(\frac{\alpha_{q}+1}{q^{\alpha_{q} / 3}}\right) .
$$

The factors on the right above are all $<1$ if $q \geq 11$, just because in that case $q^{\alpha} \geq 11^{\alpha} \geq(\alpha+1)^{3}$ for all $\alpha \geq 1$. For $q \in\{2,3,5,7\}$ and positive integers $\alpha$, we have that

$$
\frac{\alpha+1}{2^{\alpha / 3}} \leq 2, \quad \frac{\alpha+1}{3^{\alpha / 3}}<1.45, \quad \frac{\alpha+1}{5^{\alpha / 3}}<1.17, \quad \frac{\alpha+1}{7^{\alpha / 3}}<1.05 .
$$

This analysis and the fact that $2 \times 1.45 \times 1.17 \times 1.05<1.79$ shows that

$$
\tau(d)<1.79 d^{1 / 3}<1.79 p^{1 / 3} .
$$

We thus get that

$$
0.99 p<\sum_{q \leq p} \log q \leq\left(p^{1 / 2} \tau(d)+1\right) \log p<\left(1.79 p^{5 / 6}+1\right) \log p,
$$

an inequality which implies that $p<5 \cdot 10^{9}$. Finally we obtain the desired result.

Lemma 9. Lemma 8 holds for $p>5 \cdot 10^{9}$.

It remains to cover the range $\left[13,5 \cdot 10^{9}\right]$ for $p$. In a few minutes with Mathematica we compute for all $p \in[13,30000]$, that

$$
\operatorname{lcm}\left[o_{p}(q): q<p\right]>p,
$$

so we may assume that $p>30000$. In the interval $[100,1000]$ there are 27 primes numbers $q$ such that $2 q+1$ is also prime. They are the following:

$$
\begin{aligned}
& \{113,131,173,179,191,233,239,251,281,293,359,419,431, \\
& 443,491,509,593,641,653,659,683,719,743,761,809,911,953\} .
\end{aligned}
$$

Let $p>30000$ and consider one of the primes $2 q+1$ with $q$ in the above set. The order of $p$ modulo $2 q+1$ is a divisor of $2 q$, so it is 1,2 or a multiple of $q$. If it is 1 or 2 , then $q$ divides $p-1$ or $p+1$. Since $q>100$ and $p<10^{10}$, there are at most four values of $q$ for which it can be a divisor of $p-1$ and at most four values of $q$ for which it can be a divisor of $p+1$. Thus, there are at least 19 values of $q$ for which the order of $p$ modulo $q$ is a multiple of $q$. Hence,

$$
\operatorname{lcm}\left[o_{p}(q): q<p\right]>100^{19}=10^{38}>10^{10}>p,
$$

which finishes the proof. 


\section{ACKNOWLEDGEMENTS}

We thank the anonymous referee for a careful reading of this manuscript and useful comments. This work was done while all three authors were visiting the Max Planck Institute for Mathematics in Bonn in July, 2017. They thank this Institution for the invitation and excellent working conditions. In addition, B.H. and M.N. thank the RWTH Aachen, the Graduate school: Experimental and constructive algebra, chaired by G. Nebe, and the German University of Technology in Oman for its generous support. F.L. was supported in part by NRF (South Africa) Grants CPRR160325161141 and an A-rated researcher award, and by CG A (Czech Republic) Grant 17-02804S.

\section{REFERENCES}

[1] B. Heim, M. Neuhauser, and F. Rupp, Fourier coefficients of powers of the Dedekind eta function, The Ramanujan Journal, 2017, DOI: 10.1007/s11139-017-9923-4.

[2] G. Köhler, Eta Products and Theta Series Identities, Springer Monographs in Mathematics, Springer, Berlin-Heidelberg-New York (2011).

[3] M. Newman, An identity for the coefficients of certain modular forms, J. London Math. Soc., 30 (1955), 488-493.

[4] K. Ono, The Web of Modularity: Arithmetic of the Coefficients of Modular Forms and q-series, Conference Board of Mathematical Sciences 102 (2003).

[5] K. Ono and S. Robins: Superlacunary Cusp Forms. Proceedings of the AMS 123 N.4 (1995), 1021-1029.

[6] J. B. Rosser and L. Schoenfeld, Sharper bounds for the Chebyshev functions $\theta(x)$ and $\psi(x)$, Mathematics of Computation 29 (1975), 243-269.

[7] J. Serre, Sur la lacunarité des puissances de $\eta$, Glasgow Math. J. 27 (1985), 203-221.

[8] L.C. Washington, Introduction to Cyclotomic Fields, Graduate Texts in Mathematics, Springer, Berlin-Heidelberg-New York (1997).

German University of Technology in Oman, Muscat, Sultanate of Oman

Max-Planck-Institute for Mathematics, Vivatsgasse 7, 53111 Bonn, GerMANY

E-mail address: bernhard.heim@gutech.edu.om, heim@mpim-bonn.mpg.de

School of Mathematics, University of the Witwatersrand, Private Bag 3, Wits 2050, Johannesburg, South Africa

Max-Planck-Institute for Mathematics, Vivatsgasse 7, 53111 Bonn, GerMANY

Department of Mathematics, Faculty of Sciences, University of Ostrava, 30 dubna 22, 70103 Ostrava 1, Czech Republic

E-mail address: Florian.Luca@its.ac.za

German University of Technology in Oman, Muscat, Sultanate of Oman

Faculty of Mathematics, Computer Science, and Natural Sciences, RWTH Aachen University, 52056 Aachen, Germany

E-mail address: markus.neuhauser@gutech.edu.om 